interest throughout the research process. Effective guidance will need the support of researchers, funders and journals.

Discussion This research has built consensus on the need for guidance, and identified an optimal approach for assessing risk, prevention and management of conflicts of interest in interactions between population health researchers and the food industry. Further work is needed to finalise, pilot test and seek endorsement for evidence informed guidance.

\section{LB4 INDUSTRY REACTIONS TO THE UK SOFT DRINKS INDUSTRY LEVY: UNPACKING THE EVOLVING DISCOURSE FROM ANNOUNCEMENT TO IMPLEMENTATION}

TL Penney*, J Adams, M White. On behalf of the NIHR PHR SDIL Evaluation Team

\subsection{6/jech-2018-SSMabstracts.88}

Background Within the context of a global movement toward taxes on sugary drinks, the Soft Drinks Industry Levy (SDIL) is unique in its construction - a two-tiered levy that aims to encourage industry to reformulate soft drinks. Industry decisions regarding reformulation will directly influence the health impacts of the levy, however how these reactions are covered in the media will also shape a wider public discourse on sugar and health. This work will examine the evolution of industry reactions to the levy from announcement to implementation, via articles published in news media and trade press.

Methods We searched the Factiva database of UK news media and trade press. A search strategy was used to identify articles related to sugar or soft drinks and related to the levy covering March 16th 2016 to March 31st 2018. Articles were screened using predefined criteria. Analyses included: (a) description of included articles by industry actor and (b) a longitudinal, case-based, thematic analysis of each industry actor.

Results 526 articles were included covering the ongoing reactions by nine soft drinks industry actors (e.g. AG Barr, Britvic, Coca-Cola European Partners) during six policy development milestones and two national events. Early results demonstrate a discourse of disagreement with the aims of the SDIL immediately after its announcement with emergent themes including 'no evidence that sugar taxes reduce obesity', 'the poor will suffer' and 'this will destroy industry and kill jobs'. Reactions also included contradictory themes such as 'most products are not impacted' and 'we support government actions on obesity'. Throughout the consultation phase and during the Brexit vote and snap election further themes emerged including 'threats of legal action' that were not always consistent across industry actors but dominated until Royal Assent for the legislation. Throughout the parliamentary process the discourse shifted toward acceptance of the levy and undertaking efforts to adapt including 'diversification and innovative marketing efforts' and various 'cost management actions to offset the levy'. As the implementation of the levy approached, acceptance was reinforced by additional themes that sought to ensure perceived profitability with 'claims of strong sales and profits' and 'calls for investment in a sector with clear growth'.

Conclusion The shifting discourse suggests that industry actors are continually navigating issues of public, government and commercial interests, which results in conflicting narratives. Further work is needed to explore the discourses surrounding other related actors such as government, civil society and academics.

\section{\begin{tabular}{|l|l}
\hline LB5 & IS THE DURATION OF THE PRECEDING INTER-
\end{tabular} PREGNANCY INTERVAL ASSOCIATED WITH OFFSPRING'S SIZE AT BIRTH? - ANALYSIS OF A UK POPULATION-BASED COHORT}

${ }^{1} \mathrm{~N}$ Ziauddeen* ${ }^{1} \mathrm{PJ}$ Roderick, ${ }^{2} \mathrm{NS}$ Macklon, 1,3NA Alwan. ${ }^{1}$ Academic Unit of Primary Care and Population Sciences, Faculty of Medicine, University of Southampton, Southampton, UK; ${ }^{2}$ Department of Obstetrics and Gynaecology, University of Copenhagen, Zealand University Hospital, Roskilde, Denmark; ${ }^{3}$ NIHR Southampton Biomedical Research Centre, University of Southampton and University Hospital Southampton NHS Foundation Trust, Southampton, UK

\subsection{6/jech-2018-SSMabstracts.89}

Background Short and long intervals between pregnancies have been associated with increased risk of adverse birth outcomes including low birth weight and stillbirth. Birthweight is an indicator of the in-utero environment and a key early life risk factor for long-term health outcomes such as obesity and cardiovascular disease. The World Health Organization recommended in 2005 waiting at least 24 months after a live birth before getting pregnant again. There are no UK guidelines on birth spacing. We aimed to investigate the association between duration of the inter-pregnancy interval between successive live birth pregnancies and risk of having a small-for-gestational age (SGA) or large-for-gestational age (LGA) baby.

Methods A population-based cohort of prospectively collected routine healthcare data for antenatal care between January 2003 and September 2017 (total $\mathrm{n}=82098$ pregnancies) at University Hospital Southampton, Hampshire, UK was used. Records of women with their first two singleton live-birth pregnancies were analysed ( $\mathrm{n}=15922$ women). Inter-pregnancy interval was defined as timing between a live birth and the next conception. SGA was defined as $<10$ th percentile weight and LGA as >90th percentile weight for gestational age. Logistic regression was used to examine the association between risk of SGA or LGA and inter-pregnancy interval. The models were adjusted for maternal age, ethnicity, highest educational qualification, employment status, baseline maternal BMI, between pregnancy change in maternal BMI, smoking status at second pregnancy booking appointment and conception following infertility treatment. Sensitivity analyses was conducted adjusting for SGA or LGA in previous pregnancies. Results Twelve percent of first pregnancy and 7\% of second pregnancy births were SGA. Seven percent of first pregnancy and $13 \%$ of second pregnancy births were LGA. Three percent of women each had SGA and LGA babies in both pregnancies. Compared to an interval of 24-35 months, there was a lower risk of SGA birth in second pregnancy with an interval of 12-23 months (adjusted OR 0.82, 95\% CI 0.69 to $0.98, \mathrm{p}=0.03)$. The association remained after adjusting for previous outcome of SGA in sensitivity analysis. No association was observed between risk of SGA with intervals of $<12$ or $\geq 36$ months or LGA and inter-pregnancy interval. Conclusion An inter-pregnancy interval of 12-23 months was associated with lower risk of SGA, however the duration of the interval was not associated with LGA risk. In high-income countries with relatively healthy pregnant population, further research considering the potential advantages of shorter optimal interval between pregnancies than that recommended by WHO is needed. 
Acknowledgements David Cable (Electronic Patient Records Implementation and Service Manager) at University Hospital Southampton NHS Foundation Trust for support in accessing the data used in this study.

\section{Rapid fire programme}

\section{RF1 THE IMPACT OF FISCAL POLICIES ON POPULATION HEALTH AND HEALTH INEQUALITIES IN SCOTLAND: A MODELLING STUDY}

E Richardson*, A Pulford, J Parkinson, D Agbato, M Robinson. Public Health Science, NHS Health Scotland, Edinburgh, UK

\subsection{6/jech-2018-SSMabstracts.90}

Background Improving health and reducing health inequalities are important joint policy objectives. Income is a key social determinant of health, but robust evidence about the relative impacts of redistributive policies is rare. Our study aimed to estimate the potential impacts on health (premature mortality) and health inequalities of 15 fiscal policies in Scotland, including changes to income tax, council tax, and benefits, and two Universal Basic Income (UBI) schemes.

Methods EUROMOD, a detailed tax-benefit microsimulation model, was used to estimate changes in household income for each quintile of the Scottish Index of Multiple Deprivation (SIMD). Parametric survival models were used to model baseline mortality rates, and log-log models were used to estimate policy effect sizes. We estimated the impacts of each policy on premature mortality across the Scottish population after 3 years of follow up, compared to the baseline no-policy scenario, and assessed inequalities between SIMD quintiles. Data processing and modelling was conducted in R, Stata and Excel.

Results Policies predicted to both improve health and reduce health inequalities included one UBI scheme (while the other UBI scheme worsened health), replacing council tax with a local income tax, increasing Job Seeker's Allowance and Income Support, increasing tax credits, and increasing the Carer's Allowance. The health-beneficial UBI scheme would result in a $0.2 \%$ reduction in premature mortality for the whole Scottish population, a $6.1 \%$ reduction for the most deprived quintile, and a $24.7 \%$ reduction in relative inequality (as measured by the relative index of inequality). Policies that were less targeted to deprived communities either worsened health but reduced inequalities, or improved health while worsening inequalities.

Conclusion Fiscal policies have the potential for substantial effects on health and health inequalities in Scotland. The most effective policies for reducing health inequalities were those that disproportionately increased incomes in the most deprived areas. The modelling is subject to various assumptions and sources of uncertainty, but nonetheless highlights the importance of applying an inequalities lens to economic policy options.
RF2 DO PEOPLE IN MORE DEPRIVED AREAS HAVE A HIGHER RISK OF ALCOHOL-RELATED HOSPITAL ADMISSION, AFTER ACCOUNTING FOR INDIVIDUALLY RECORDLINKED DATA ON ALCOHOL CONSUMPTION AND SMOKING?

${ }^{1}$ A Gartner*, 'S Paranjothy, 'L Trefan, ${ }^{2} S$ Moore, ${ }^{3}$ A Akbari, ${ }^{4} J$ Kennedy, 'D Fone, 'D Farewell. 'Division of Population Medicine, School of Medicine, Cardiff University, Cardiff, UK; ${ }^{2}$ Alcohol and Violence Research Group/Crime and Security Research Institute, Cardiff University, Cardiff, UK; ${ }^{3}$ Farr Institute, Swansea University Medical School, Swansea University, Swansea, UK; ${ }^{4}$ Swansea University Medical School, Swansea University, swansea, UK

\subsection{6/jech-2018-SSMabstracts.91}

Background Greater area deprivation is associated with a higher risk of alcohol-related harm. Few studies have investigated longitudinal patterns of harm using record-linked alcohol consumption, and none considered drink type which is associated with deprivation. This study aims to investigate whether the type of drink is associated with the observed higher risk of alcohol-related hospital admission (ARHA) in people living in deprived areas.

Methods A total of 11229 people aged 16 and over responded to the Welsh Health Survey in 2013 and 2014, consenting to data linkage. Responses were record-linked within the Secure Anonymised Information Linkage Databank (SAIL) to wholly attributable ARHA (defined by Public Health England) 8 years before the survey month until the end of 2016. They were censored for death or leaving Wales using the Welsh Demographic Service. To each lower super output area (LSOA) at survey month we linked the Welsh Index of Multiple Deprivation 2011, grouping the two more deprived quintiles and three less deprived quintiles. Alcohol consumption and smoking status throughout the study period were estimated from survey responses.

We estimated hazard ratios (HR) with 95\% confidence intervals $(95 \% \mathrm{CI})$ for the risk of (multiple) ARHA for deprivation groups using age-based recurrent-event models. The study period started 3 years before the survey. The first model adjusted for sex, time since the last and number of historic ARHA during 5 years before study start. The second model also adjusted for the number of units reported by drink type (beer and cider; wine and champagne; spirits including alcopops) on the heaviest drinking day in the past week and smoking status.

Results 131 respondents had at least one ARHA. People living in more deprived areas had a higher risk of ARHA (HR 1.52; 95\% CI 1.08 to 2.14) compared to less deprived. In model 2, adjustment for units of alcohol drunk and smoking reduced the risk of ARHA for more deprived areas (HR 1.29; 95\% CI 0.90 to 1.84 ) with smoking and historic admission having particularly strong effects. Unit increases of spirits drunk were positively associated with increasing risk of ARHA (HR 1.05; 95\% CI 1.01 to 1.10 ), higher than for other drink types.

Conclusion Respondents living in more deprived areas had only a slightly higher risk of alcohol-related hospital admission, considering similar unit consumption, smoking and historic admission. Although significant, adjusting for units by type of drink did not markedly change the socioeconomic pattern of alcohol-related harm. 